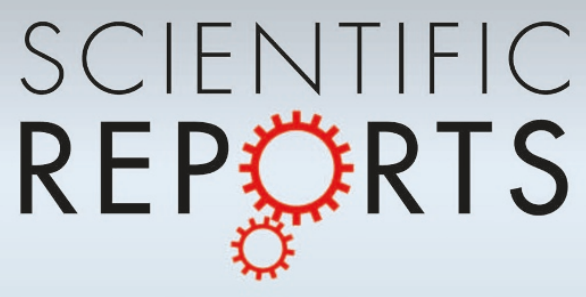

\title{
OPEN A monomer-trimer model supports intermittent glucagon fibril growth
}

SUBJECT AREAS:

KINETICS

PROTEINS

COMPUTATIONAL BIOPHYSICS

CONFOCAL MICROSCOPY

Received

20 October 2014

Accepted

19 January 2015

Published

11 March 2015

Correspondence and requests for materials should be addressed to A.K. (andrej@physics. harvard.edu); L.B.O. (oddershede@nbi.dk) or M.H.J. (mhjensen@ nbi.dk)

* Current address: University of Cambridge,

Department of

Chemical Engineering and Biotechnology,

Cambridge CB2 3RA,

UK.

\author{
Andrej Košmrli' , Pia Cordsen², Anders Kyrsting ${ }^{2 *}$, Daniel E. Otzen ${ }^{2,3}$, Lene B. Oddershede ${ }^{2}$ \\ \& Mogens $\mathrm{H}$. Jensen ${ }^{2}$
}

\begin{abstract}
${ }^{1}$ Harvard University, Department of Physics, 17 Oxford Street, Cambridge, MA 02138 , USA, ${ }^{2}$ Copenhagen University, Niels Bohr Institute, CMOL, Blegdamsvej 17, DK-2 100 Copenhagen, Denmark, ${ }^{3}$ Interdisciplinary Nanoscience Center (iNANO), Department of Molecular Biology and Genetics, Aarhus University, Gustav Wieds Vej 14, DK-8000 Aarhus C, Denmark.
\end{abstract}

We investigate in vitro fibrillation kinetics of the hormone peptide glucagon at various concentrations using confocal microscopy and determine the glucagon fibril persistence length $60 \mu \mathrm{m}$. At all concentrations we observe that periods of individual fibril growth are interrupted by periods of stasis. The growth probability is large at high and low concentrations and is reduced for intermediate glucagon concentrations. To explain this behavior we propose a simple model, where fibrils come in two forms, one built entirely from glucagon monomers and one entirely from glucagon trimers. The opposite building blocks act as fibril growth blockers, and this generic model reproduces experimental behavior well.

M isfolding and aggregation of peptides and proteins into fibrils are the hallmarks of around 40 human diseases $^{1,2}$. Understanding the fibrillation process of one protein may provide a generic mechanistic insight useful for understanding fibrillation of a class of proteins. In this paper we focus on the protein glucagon, which is a 29 amino acid residue hormone peptide, that upregulates blood sugar levels. It is an important pharmaceutical molecule, which is used to treat diabetic patients in situations of acute hypoglycemia $\mathrm{T}^{3,4}$. As obesity and the number of diabetic patients is increasing, this drug becomes more and more relevant. The active state of glucagon is the monomer, but during pharmaceutical production the peptide has a high tendency to misfold and aggregate into fibrils devoid of biological function ${ }^{5}$. When glucagon is solubilized, it can be found in two states, which produce glucagon fibrils of different morphologies. Below a concentration of $1 \mathrm{mg} / \mathrm{mL}$, glucagon is predominantly found in an unstructured monomeric state, while above $1 \mathrm{mg} / \mathrm{mL}$ glucagon form associated states such as trimers and other oligomers ${ }^{6-10}$. The monomer and oligomer precursor states lead to twisted and non-twisted fibrils, respectively ${ }^{11-13}$. Experiments suggest that at high glucagon concentrations, the monomeric species are not incorporated into fibrils ${ }^{10}$ and the growth of twisted fibrils is inhibited ${ }^{12}$.

Fibrillation of proteins and peptides is typically followed in bulk using the fibril-binding fluorescent dye Thioflavin $\mathrm{T}(\mathrm{ThT})$. While ThT-based fibrillation kinetics can provide highly valuable information on the mechanisms of fibrillation ${ }^{14}$, studies of the growth of individual fibrils can also yield important insights. This information is provided by techniques such as Total Internal Reflection Fluorescence Microscopy (TIRFM) and Confocal Microscopy (CM). In TIRFM the observation depth is $\sim 150 \mathrm{~nm}$ while with CM it is $\sim 500 \mathrm{~nm}$. Another elegant way to resolve fibers is by propelling a nanoparticle along the fibre ${ }^{15}$.

Previously, we have studied growth of individual glucagon fibrils in real-time using TIRFM $^{16}$ at one fixed glucagon concentration. In that study, fibril growth was found to be interrupted by periods of stasis, and the statistics of growth and stasis durations were well described by a Poissonian process. This dynamic behaviour was denoted stop-go kinetics. Switching rates between the growing and arrested states suggested the probability of being in the growing state to be $\sim 1 / 4$. To explain this value, a Markovian four-state model of fibril growth was proposed. The model predicted that the growth probability is independent of the glucagon concentration. This is in contrast to our findings since here we demonstrate that the fibril growth probability does depend on the glucagon concentration.

Here we significantly expand our previous work ${ }^{16}$ by monitoring fibril kinetics over a wide range of glucagon concentrations and by proposing a new model that captures the underlying molecular mechanisms of the process. This allows us to sample conditions spanning different precursor states of glucagon, i.e. monomers or trimers, leading to twisted or non-twisted fibrils, respectively. Fibrils were labeled with the fluorescent dye ThT and monitored using a confocal microscope with an Argon laser. On freshly plasmated glass plates we observed a volume of $\sim 40 \times 40 \times 0.5 \mu \mathrm{m}^{3}$. For each of the five different initial glucagon concentrations $(1.5,3,6,10$ and 
$15 \mathrm{mg} / \mathrm{mL}$ ), a minimum of two experiments were conducted in aqueous buffer (50 $\mathrm{mM}$ glycine $\mathrm{HCl}, \mathrm{pH} 2.5)$. The time interval between captured frames was 3.3 mins and the total observation time of each experiment was about three days. When fibrils grew along the surface we tracked their length as a function of time. Sample images of real time growth of an individual fibril are shown in Fig. 1(a-c). The observed growing fibrils are relatively straight and their persist-
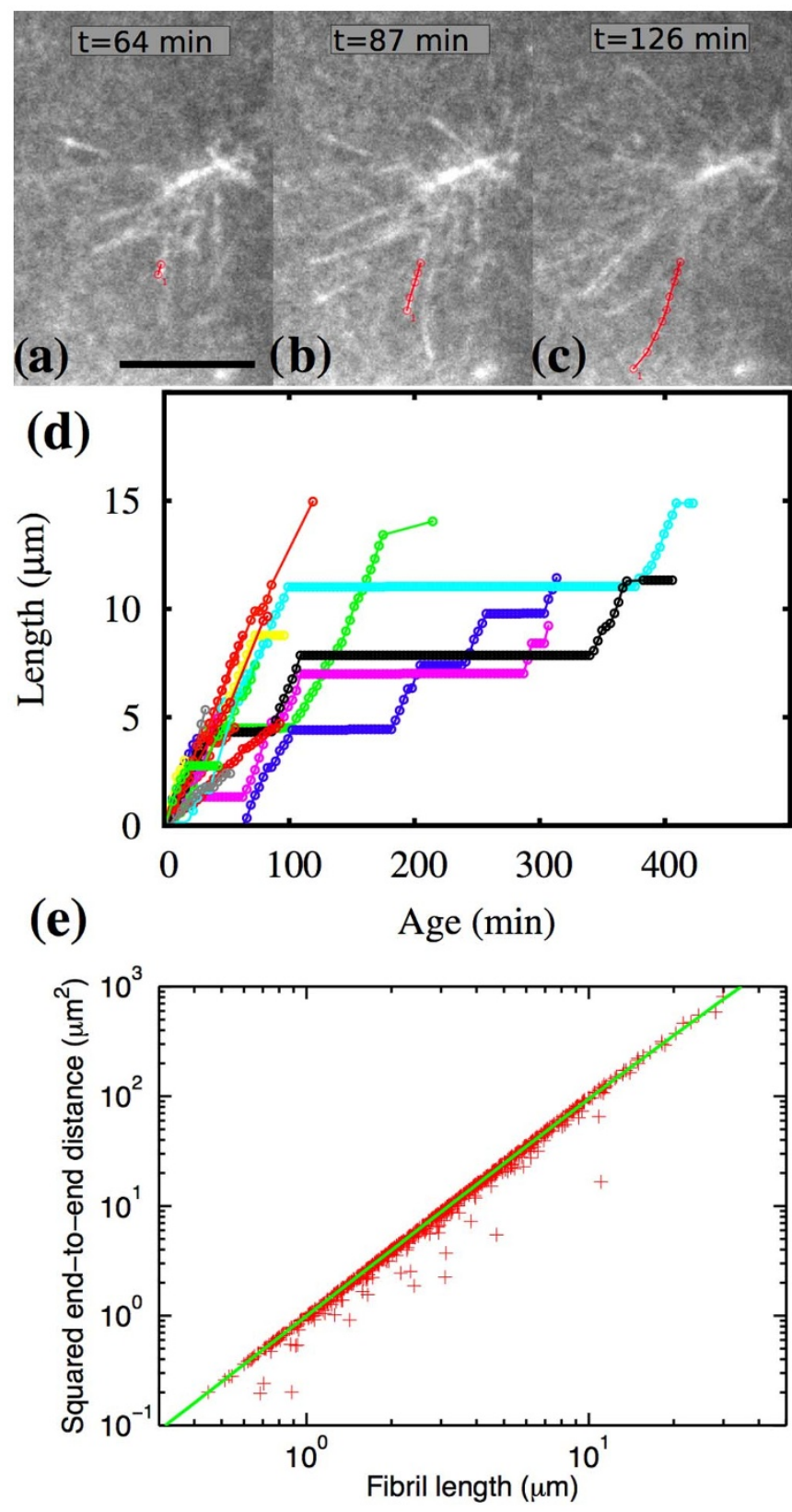

Figure $1 \mid(a-c)$ : Confocal microscopy images of glucagon fibrils with initial concentration $3 \mathrm{mg} / \mathrm{mL}$ in aqueous buffer $(50 \mathrm{mM}$ glycine $\mathrm{HCl}, \mathrm{pH}$ 2.5) at three consecutive times: 64, 87 and 126 mins after the onset of fibrillation. Scale bar shows $5 \mu \mathrm{m}$. Each circle represents a data point and the red line represents the cumulated tracked positions of the growing fibril end. (d) Growth of 20 fibrils at the glucagon concentration of $3 \mathrm{mg} / \mathrm{mL}$. Plateaus correspond to arrested states while fibrils elongate outside the plateaus. (e) The average end-to-end-distance squared $\left(\left\langle R_{e e}^{2}\right\rangle\right)$ as a function of fibril length. The solid green line is obtained by fitting Eq. (1) to combined experimental data (red points) from all glucagon concentrations. The persistence length of fibrils is returned by the fit as 60 $\pm 2 \mu \mathrm{m}$. (a)

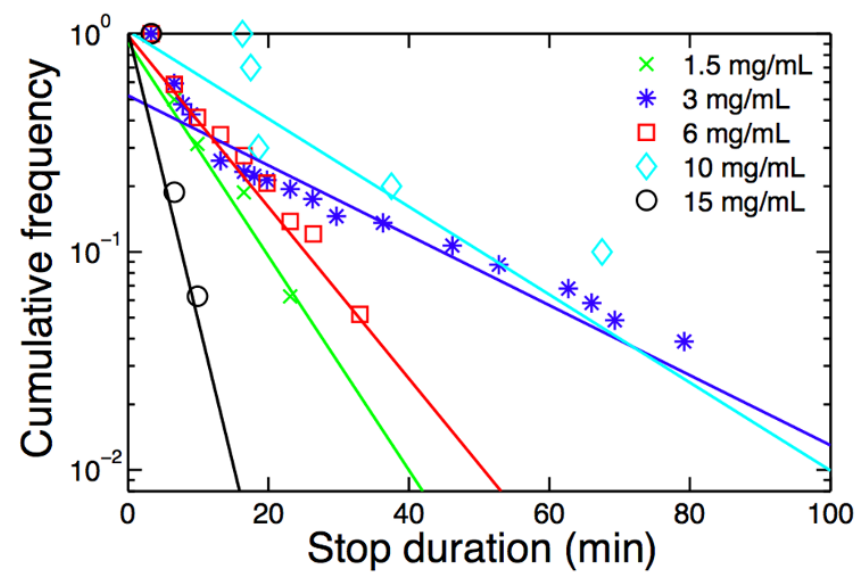

(b)

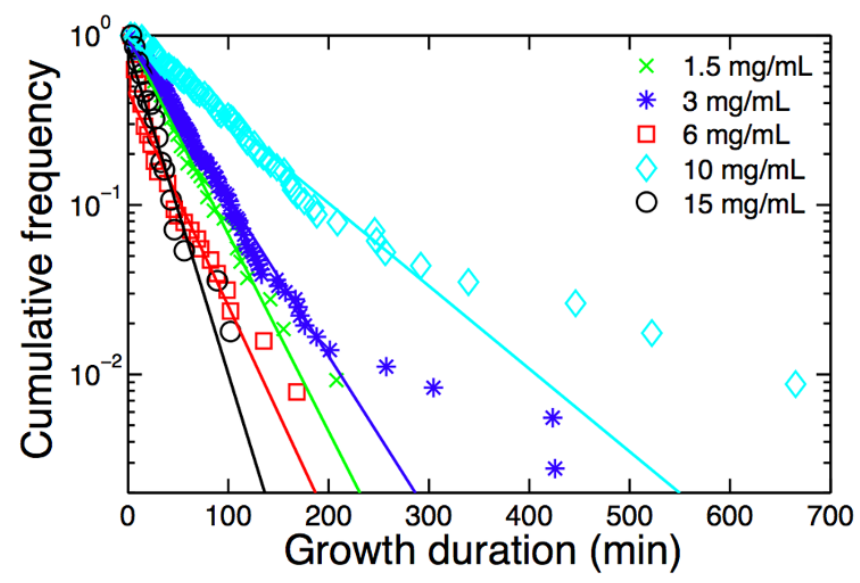

Figure $2 \mid$ Distributions of stop (a) and growth (b) durations for fibrils grown at various glucagon concentrations. Straight lines indicate linear fits to the cumulative data. Three extremely long pauses were removed from the $3 \mathrm{mg} / \mathrm{mL}$ sample.

ence length $\ell_{p}$ can be extracted by comparing the geometric distance between fibril ends $R_{e e}$ to the fibril length $L$. For semi-flexible fibrils the average end-to-end distance is expected to $\mathrm{be}^{17}$

$$
\left\langle R_{e e}^{2}\right\rangle=2 \ell_{p} L-2 \ell_{p}^{2}\left[1-e^{-L / \ell_{p}}\right]
$$

which agrees extremely well with experimental data (Fig. 1e). The fitting of equation above to experimental data provides a persistence length $\ell_{p}=60 \pm 2 \mu \mathrm{m}$. Note that this is of the same order as the persistence length of actin filaments $(\sim 20 \mu \mathrm{m})^{18}$, while much smaller than the persistence length of microtubules $(\sim 5,000 \mu \mathrm{m})^{18}$, and larger than the persistence lengths of DNA $(\sim 50 \mathrm{~nm})^{19}$ and amyloid fibrils $(0.1-4 \mu \mathrm{m})^{20}$.

By inspecting the time courses of fibril lengths (Fig. 1d), we find that at all glucagon concentrations the fibril growth is characterized by periods of growth (go state) interrupted by periods of stasis (stop state). The stop states are seen as plateaus, where the fibril does not elongate. As seen in our previous work ${ }^{16}$, the distributions of the stop and go event durations (displayed in Fig. 2) follow exponential distributions and are fitted to the form $f(x)=a \cdot \exp (-k \cdot t)$. A fibril leaves the stop state at rate $k_{\mathrm{s} \rightarrow \mathrm{g}}$ given by stop durations (Fig. 2a) and go state at rate $k_{\mathrm{g} \rightarrow \mathrm{s}}$ given by growth durations (Fig. 2b). Both switching rates depend on the glucagon concentration and results are summarized in Table 1. 
Table 1 | Values of switching rates between growth and stop states obtained by fitting experimental data in Fig. 2 and the corresponding probability of growth as defined in Eq. (16)

\begin{tabular}{lccc}
{$\left[G_{\text {tot }}\right](\mathrm{mg} / \mathrm{mL})$} & $k_{\mathrm{s} \rightarrow \mathrm{g}}\left(\mathrm{min}^{-1}\right)$ & $k_{\mathrm{g} \rightarrow \mathrm{s}}\left(\mathrm{min}^{-1}\right)$ & $p_{G}$ \\
\hline 1.5 & $0.113 \pm 0.025$ & $0.0268 \pm 0.0005$ & $0.808 \pm 0.034$ \\
3 & $0.037 \pm 0.002$ & $0.0215 \pm 0.0003$ & $0.632 \pm 0.015$ \\
6 & $0.091 \pm 0.006$ & $0.0292 \pm 0.0012$ & $0.756 \pm 0.014$ \\
10 & $0.046 \pm 0.020$ & $0.0113 \pm 0.0002$ & $0.805 \pm 0.069$ \\
15 & $0.306 \pm 0.096$ & $0.0444 \pm 0.0024$ & $0.873 \pm 0.035$ \\
\hline
\end{tabular}

The access to kinetic data at different glucagon concentrations allows us to develop a model for glucagon's fibrillation. The analytical models for the kinetics of fibril growth were initiated with the Oosawa model ${ }^{21}$ and further elaborated to include hydrolysis and breakage of fibrils ${ }^{22,23}$. Our model is an extension of the Oosawa model, which includes both monomers and trimers as basic building blocks for fibrils.

To explain the intermittent fibril growth behavior we propose a model sketched in Fig. 3. In the bulk solution glucagon monomers are in equilibrium with glucagon trimers and these two components give rise to twisted and non-twisted fibrils, respectively. Successive binding of glucagon monomers to the twisted fibril end corresponds to the growing state, while binding of trimers to the twisted fibril end prevents further growth until the trimer is detached. During this time the twisted fibril appears to be in the arrested state. The opposite is true for non-twisted fibrils, which are formed from glucagon trimers, while glucagon monomers inhibit their growth.

In the mean field approximation, the fibril growth probability can be expressed in terms of the model rate constants, which are defined in Fig. 3, and compared to the experimentally observed growth probabilities. Below we use simple physical terms to guide the derivation of main equations and to interpret results at various levels of glucagon concentrations. We tried to be systematic in naming rate constants (see Fig. 3): superscripts $(M)$ and $(T)$ are used to describe monomer and trimer fibrils, respectively; subscripts $b, u$ and $r$ are used to describe binding, unbinding and rearrangement events, respectively; and subscripts 1 and 3 are used to describe binding and unbinding of glucagon monomer and trimers, respectively. The growth probability predicted by the model is calculated by considering the average time spent in the growing or arrested state as outlined below. In a bulk solution glucagon is in equilibrium between monomers $(\mathrm{M})$ of concentration $[G]$ and trimers $(\mathrm{T})$ of concentration $\left[G_{3}\right]$ with the equilibrium constant

$$
K_{0}^{2}=\frac{[G]^{3}}{\left[G_{3}\right]}=\frac{k_{31}}{k_{13}}
$$

and the total glucagon concentration $\left[G_{\mathrm{tot}}\right]=[G]+3\left[G_{3}\right]$. As mentioned before at low (high) glucagon concentrations, i.e., $\left[G_{\text {tot }}\right] \ll K_{0}\left(\left[G_{\text {tot }}\right] \gg K_{0}\right)$, glucagon is predominantly in the monomer (trimer) state.

For the free growing twisted fibril end it takes on average the time $\left(k_{b 1}^{(M)}[G]+k_{b 3}^{(M)}\left[G_{3}\right]\right)^{-1}$ before the glucagon monomer or trimer binds to the tip. This occurs with probabilities $p_{1}^{(M)}$ or $p_{3}^{(M)}$ respectively, where

$$
p_{1}^{(M)}=\frac{k_{b 1}^{(M)}[G]}{\left(k_{b 1}^{(M)}[G]+k_{b 3}^{(M)}\left[G_{3}\right]\right)}=1-p_{3}^{(M)} .
$$

If a glucagon monomer is bound to the growing twisted fibril end, it takes on average the time $\left(k_{u 1}^{(M)}+k_{r}^{(M)}\right)^{-1}$ for the glucagon monomer to unbind with probability $p_{1 u}^{(M)}$ or to undergo conformational rearrangement and form a longer fibril with probability $p_{1 g}^{(M)}$, where

$$
p_{1 u}^{(M)}=\frac{k_{u 1}^{(M)}}{k_{r}^{(M)}+k_{u 1}^{(M)}}=1-p_{1 g}^{(M)} .
$$

\section{Low concentration High concentration}

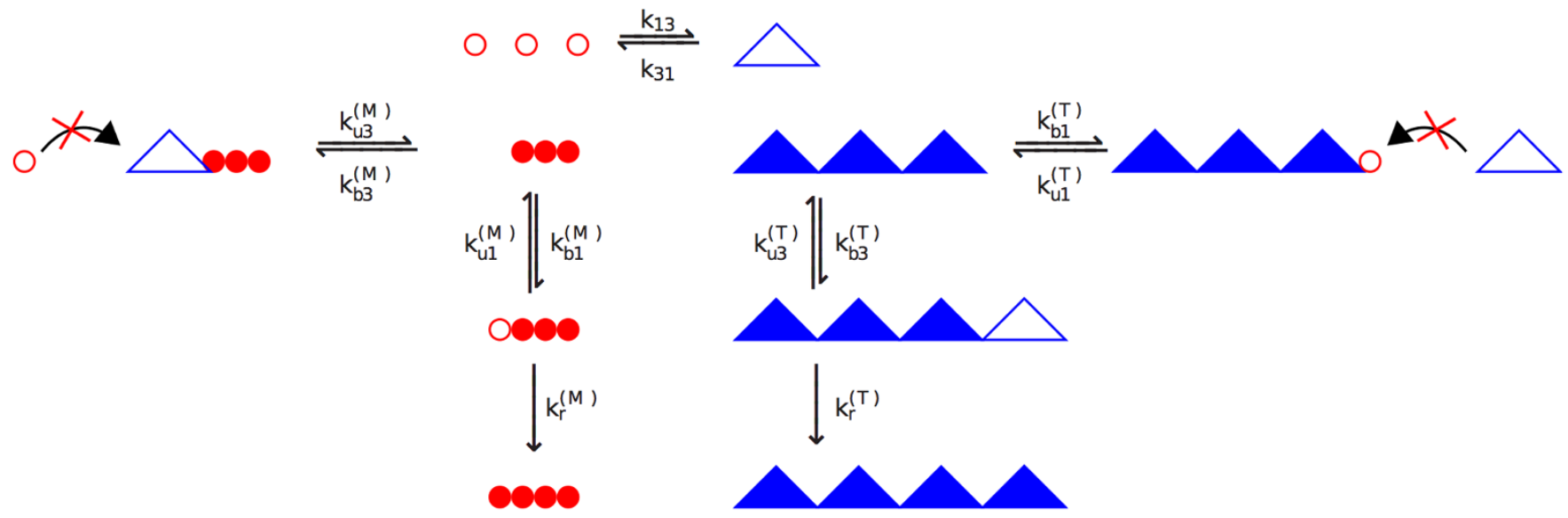

Figure 3 Schematic overview of the glucagon growth model showing (upper part) monomer-trimer equilibrium and (lower part) fibrillation process. Glucagon monomers are in equilibrium with glucagon trimers. Elongation of a fibril is a two-step process, which can be interrupted by binding of the other oligomer. Fibrils consist of either monomers or trimers but never a combination of the two. A glucagon trimer (monomer) can bind to a growing fibril end and then dissociate or elongate the fibril after conformational rearrangement. Filled triangles (circles) symbolize trimers (monomers) bound irreversibly to a fibril, while hollow triangles (circles) mean unbound trimers (monomers). A glucagon monomer (trimer) can also bind to a growing fibril end, but in this arrested state it prevents further attachment of glucagon trimers (monomers). 
The average time $\tau_{1}^{(M)}$ for a monomer to bind and subsequently either unbind or undergo conformational rearrangement to elongate the twisted fibril is

$$
\tau_{1}^{(M)}=\frac{1}{\left(k_{b 1}^{(M)}[G]+k_{b 3}^{(M)}\left[G_{3}\right]\right)}+\frac{1}{\left(k_{u 1}^{(M)}+k_{r}^{(M)}\right)},
$$

while the average time $\tau_{3}^{(M)}$ for the binding and unbinding of a glucagon trimer is

$$
\tau_{3}^{(M)}=\frac{1}{\left(k_{b 1}^{(M)}[G]+k_{b 3}^{(M)}\left[G_{3}\right]\right)}+\frac{1}{k_{u 3}^{(M)}} .
$$

We define the growth probability $p_{G}^{(M)}$ as the expected average fraction of time the twisted fibril spends in the growing state:

$$
p_{G}^{(M)}=\frac{p_{1}^{(M)} p_{1 g}^{(M)} \tau_{1}^{(M)}}{p_{1}^{(M)} \tau_{1}^{(M)}+p_{3}^{(M)} \tau_{3}^{(M)}} .
$$

Similarly, we can analyze the dynamics of the growing non-twisted fibrils, which are formed from glucagon trimers. The growth probability for non-twisted fibrils is then

$$
p_{G}^{(T)}=\frac{p_{3}^{(T)} p_{3 g}^{(T)} \tau_{3}^{(T)}}{p_{1}^{(T)} \tau_{1}^{(T)}+p_{3}^{(T)} \tau_{3}^{(T)}},
$$

where all quantities are defined in analogous way as above for the twisted fibrils. However, in this case the role of glucagon monomers and trimers is reversed, i.e., in Eqns. (3-6) above one should replace $(M)$ with $(T)$ and make the $1 \leftrightarrow 3$ substitutions to obtain the relevant quantities. Since the number of twisted and non-twisted fibrils is proportional to the number of glucagon monomers and trimers, respectively, the probability $p_{G}$ that the randomly chosen fibril is found in the growing state is

$$
p_{G}=\frac{p_{G}^{(M)}[G]+p_{G}^{(T)}\left[G_{3}\right]}{[G]+\left[G_{3}\right]} .
$$

It is possible to derive the exact expression for the growth probability above in terms of the rate constants and the total glucagon concentration, but for simplicity we present only the asymptotic regimes at low and high glucagon concentration. At low glucagon concentration, $\left[G_{t o t}\right] \ll K_{0}$, the majority of glucagon is in the monomeric state. The slow time scales correspond to binding of glucagon monomers or trimers to the fibril ends and the growing probability for twisted fibrils is

$$
p_{G}^{(M)} \approx \frac{k_{r}^{(M)}}{\left(k_{u 1}^{(M)}+k_{r}^{(M)}\right)}\left[1-\frac{k_{b 3}^{(M)}}{k_{b 1}^{(M)}}\left(\frac{\left[G_{t o t}\right]}{K_{0}}\right)^{2}\right] .
$$

There are only a small number of non-twisted fibrils, whose growth is further suppressed by binding of glucagon monomers

$$
p_{G}^{(T)} \approx \frac{k_{b 3}^{(T)} k_{r}^{(T)}}{k_{b 1}^{(T)}\left(k_{u 3}^{(T)}+k_{r}^{(T)}\right)}\left(\frac{\left[G_{t o t}\right]}{K_{0}}\right)^{2} .
$$

The fibril growth probability is thus approximately

$$
p_{G}^{\text {low }} \approx \frac{p_{G}^{(M)}[G]}{\left([G]+\left[G_{3}\right]\right)}
$$

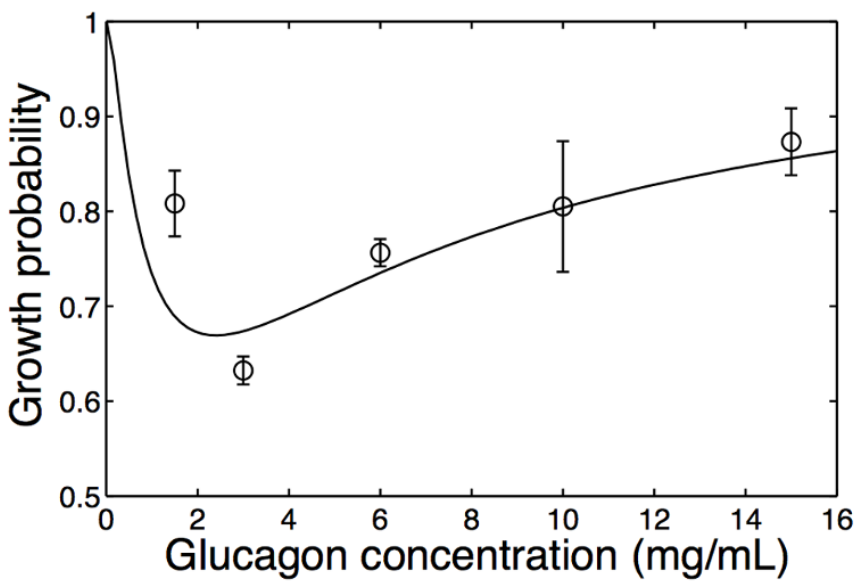

Figure $4 \mid$ Experimentally measured fibril growth probabilities (black bars) calculated from Eq.(16). The error bars in experimentally observed growth probabilities are calculated from uncertainties in the switching rates between the stop and go states from fitting in Figs. 2(a-b). The black line shows a fit of the model in Eq. (9) to the experimental data.

where $[G] /\left([G]+\left[G_{3}\right]\right) \approx 1-\left[G_{\mathrm{tot}}\right]^{2} / K_{0}^{2}$.

At high glucagon concentrations, $\left[G_{t o t}\right] \gg K_{0}$, most of the glucagon is in the trimeric state. The binding events are fast because of the large concentration of glucagon trimers and the slow time steps are the unbinding and conformational reconfiguration. The growth probability of non-twisted fibrils is approximately

$$
p_{G}^{(T)} \approx \frac{k_{r}^{(T)}}{\left(k_{u 3}^{(T)}+k_{r}^{(T)}\right)}-\frac{k_{b 1}^{(T)} k_{r}^{(T)}}{k_{b 3}^{(T)} k_{u 1}^{(T)}}\left(\frac{3 K_{0}}{\left[G_{t o t}\right]}\right)^{2 / 3} .
$$

There are only a small number of twisted fibrils, whose growth is further suppressed by binding of glucagon trimers

$$
p_{G}^{(M)} \approx \frac{k_{b 1}^{(M)} k_{r}^{(M)} k_{u 3}^{(M)}}{k_{b 3}^{(M)}\left(k_{u 1}^{(M)}+k_{r}^{(M)}\right)^{2}}\left(\frac{3 K_{0}}{\left[G_{t o t}\right]}\right)^{2 / 3} .
$$

The fibril growth probability is thus approximately

$$
p_{G}^{\text {high }} \approx \frac{p_{G}^{(T)}\left[G_{3}\right]}{\left([G]+\left[G_{3}\right]\right)},
$$

where $\left[G_{3}\right] /\left([G]+\left[G_{3}\right]\right) \approx 1-\left(3 K_{0} /\left[G_{\text {tot }}\right]\right)^{2 / 3}$.

Table 2 | Values of unknown rate constants obtained by fitting experimental data in Fig. 4. The value of equilibrium constant $K_{0}=\sqrt{k_{31} / k_{13}} \approx 1 \mathrm{mg} / \mathrm{mL}$ is known experimentally ${ }^{12}$. The rate constants whose fitted ratios are given in this Table are defined in Figure 3

\begin{tabular}{llll}
$\frac{k_{b 3}^{(M)}}{k_{b 1}^{(M)}}$ & 0.88 & $\frac{k_{b 1}^{(T)}}{k_{b 3}^{(T)}}$ & 2.9 \\
$\frac{k_{u 3}^{(M)}}{k_{b 1}^{(M)} K_{0}}$ & $7.0 \times 10^{7}$ & $\frac{k_{u 1}^{(T)}}{k_{b 3}^{(T)} K_{0}}$ & $1.8 \times 10^{8}$ \\
$\frac{k_{u 1}^{(M)}}{k_{b 1}^{(M)} K_{0}}$ & $6.4 \times 10^{-9}$ & $\frac{k_{u 3}^{(T)}}{k_{b 3}^{(T)} K_{0}}$ & $8.5 \times 10^{-9}$ \\
$\frac{k_{r}^{(M)}}{k_{b 1}^{(M)} K_{0}}$ & 0.59 & $\frac{k_{r}^{(T)}}{k_{b 3}^{(T)} K_{0}}$ & 1.5 \\
\hline
\end{tabular}




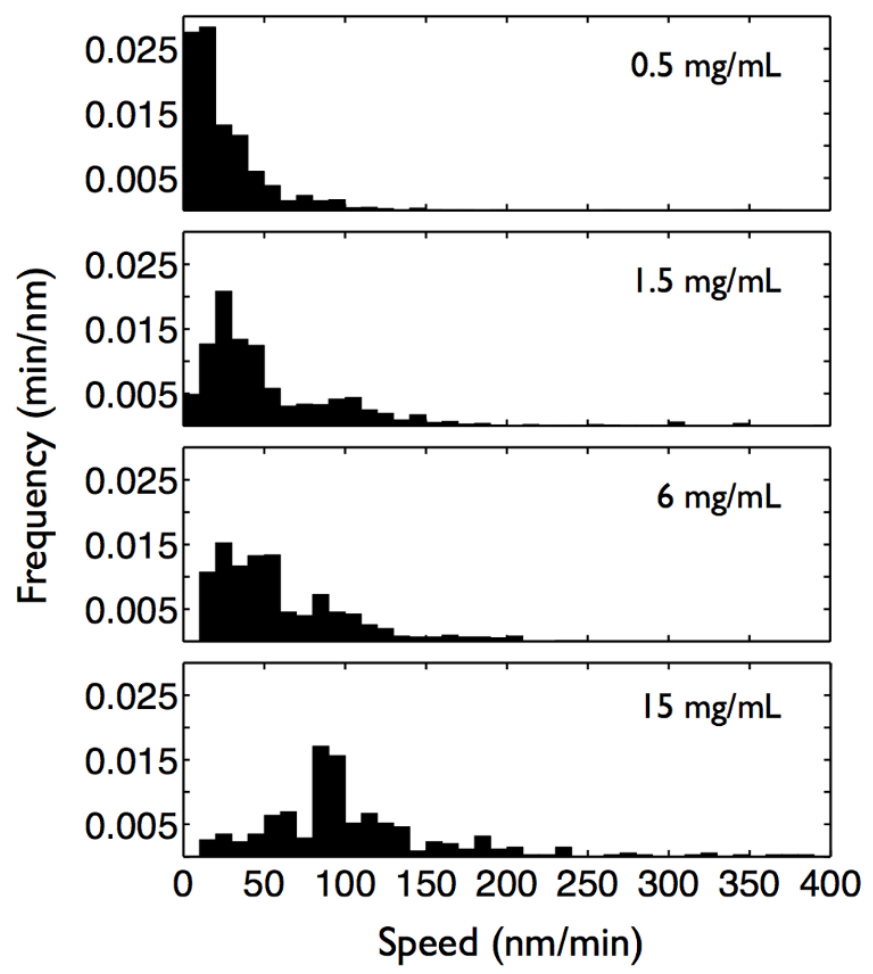

Figure 5 | Growth speed distributions of glucagon fibrils at various glucagon concentrations (displayed in top-right corners) are presented as histograms (boxes of width $10 \mathrm{~nm} / \mathrm{min}$ ).

At intermediate glucagon concentrations, $\left[G_{t o t}\right] \sim K_{0}$, there is a mix of twisted and non-twisted fibrils whose growth is suppressed due to binding of the opposite glucagon components.

The probability that at any moment a given fibril is in the growing state can be determined from the experimental switching rates between the stop and go states as

$$
p_{G}=\frac{k_{\mathrm{s} \rightarrow \mathrm{g}}}{k_{\mathrm{s} \rightarrow \mathrm{g}}+k_{\mathrm{g} \rightarrow \mathrm{s}}} .
$$

Assuming that the error estimates $\sigma_{\mathrm{s} \rightarrow \mathrm{g}}$ and $\sigma_{\mathrm{g} \rightarrow \mathrm{s}}$ for experimental switching rates $k_{\mathrm{s} \rightarrow \mathrm{g}}$ and $k_{\mathrm{g} \rightarrow \mathrm{s}}$, which are obtained by linear fits in Fig. 2, are uncorrelated, we can estimate the error $\sigma_{G}$ for the growth probability as

$\sigma_{G}^{2}=\left(\frac{\partial p_{G}}{\partial k_{\mathrm{g} \rightarrow \mathrm{s}}}\right)^{2} \sigma_{\mathrm{g} \rightarrow \mathrm{s}}^{2}+\left(\frac{\partial p_{G}}{\partial k_{\mathrm{s} \rightarrow \mathrm{g}}}\right)^{2} \sigma_{\mathrm{s} \rightarrow \mathrm{g}}^{2}=\frac{k_{\mathrm{s} \rightarrow \mathrm{g}}^{2} \sigma_{\mathrm{g} \rightarrow \mathrm{s}}^{2}+k_{\mathrm{g} \rightarrow \mathrm{s}}^{2} \sigma_{\mathrm{s} \rightarrow \mathrm{g}}^{2}}{\left(k_{\mathrm{s} \rightarrow \mathrm{g}}+k_{\mathrm{g} \rightarrow \mathrm{s}}\right)^{4}}$

The measured fibril growth probabilities at different glucagon concentrations, given by equation (16) above, are displayed in Fig. 4 as black bars (see also Table 1). We notice that fibril growth probabilities are large at high and low glucagon concentrations, while they are smaller at intermediate glucagon concentrations $(\sim 3 \mathrm{mg} / \mathrm{mL})$, which approximately correspond to the equilibrium constant $K_{0}$ of glucagon monomers and trimers ${ }^{12}$. In order to see how our model compares to the experimental data, we need to determine 12 rate constants (see Fig. 3). However, if we are only interested in the growth probability of fibrils there are 9 independent constants, because the growth probability does not depend on the absolute values of time scales for growing fibrils and the time scale for switching between monomers and trimers. In practice we fixed values of $k_{13}, k_{b 1}^{(M)}$ and $k_{b 3}^{(T)}$. Since the value of equilibrium constant $K_{0} \approx$ $1 \mathrm{mg} / \mathrm{ml}$ is known experimentally ${ }^{12}$, we fit the ratios of the other 8 rate constants to the 5 data points in Fig. 4 (see also Table 2). Model fits quite well to the experimental data and results suggest that once the correct component binds to the growing fibril end, then the rearrangement process leading to growth is much more likely than unbinding $\left(k_{r}^{(M)} \gg k_{u 1}^{(M)}\right)$ and $k_{r}^{(T)} \gg k_{u 3}^{(T)}$. Fitting also suggests that once the wrong component binds to the growing fibril end, then it unbinds very quickly $\left(k_{r}^{(M)} \ll k_{u 3}^{(M)}\right)$ and $k_{r}^{(T)} \ll k_{u 1}^{(T)}$. Previous study of glucagon fibrillation at a very low concentration $(0.25 \mathrm{mg} / \mathrm{mL})$ found the growth probability to be $\sim 1 / 4^{16}$, which is smaller than the growth probabilities observed in our experiments (Fig. 4). We speculate that in that study, fibril seeds grown at a higher glucagon concentration could bias the distribution of fibrils towards trimeric fibrils and hence result in a lower growth probability than predicted by our equilibrium model.

The model presented above with two competing fibril morphologies is further supported by the measurements of speeds at which the fibrils are growing (Fig. 5). The speed distributions seem to have two peaks, whose magnitudes depend on the glucagon concentration. At low glucagon concentration the dominant peak is at $\sim 20-30 \mathrm{~nm} /$ min, which probably corresponds to the growing speed of twisted fibrils composed of glucagon monomers. On the other hand, at large glucagon concentration the dominant peak is at $\sim 100 \mathrm{~nm} / \mathrm{min}$, which probably corresponds to the growing speed of non-twisted fibrils composed of glucagon trimers.

In conclusion, we present a monomer-trimer model for glucagon fibrillation and compared it with our experimental data. The model predicts a concentration dependent growth probability, which we test experimentally at various glucagon concentrations by analyzing the distributions of growth and stasis duration. Our model captures the short time behavior of growth and pause durations and reproduce the experimentally observed growth probability well. The stopgo kinetics observed requires two contrasting precursor states, one of which elongates while the other one blocks. Thus, the model might generically also explain, e.g., fibril growth kinetics for $\beta$-lactoglobulin which exists in a monomer-dimer equilibrium, where only the monomer is capable of elongating fibrils (via a partially unfolded state $)^{24}$. The model is an expansion of current models of amyloid fibril growth, which only use a single precursor species that feeds into the fibril, though this precursor species may undergo multiple conformational changes before it reaches a state that is activated for incorporation into the fibrillar structure ${ }^{2,25}$. This type of selective uptake of precursors from a pool of different species may also be relevant for other types of assembly, such as microtubule polymerization/depolymerization and actin filament growth. Actin is known to access different conformational states under physiological conditions ${ }^{26}$. This may allow the growing filaments to select the most appropriate state for incorporation as well as leading to "structural plasticity" in the polymer ${ }^{27}$. On the other hand, our model is not likely to be relevant for more limited assemblies such as viral capsids, which consist of a finite number of capsid proteins and therefore does not grow indefinitely; furthermore the precursor capsid proteins do not populate different conformational states ${ }^{28}$.

1. Selkoe, D. J. Folding proteins in fatal ways. Nature 426, 900-904 (2003).

2. Chiti, F. \& Dobson, C. M. Protein misfolding, functional amyloid, and human disease. Annu. Rev. Biochem. 75, 333-366 (2006).

3. Drucker, D. J. Biologic actions and therapeutic potential of the proglucagonderived peptides. Nat. Clin. Pract. Endocrinol. Metab. 1, 22-31 (2005).

4. Jiang, G. \& Zhang, B. B. Glucagon and regulation of glucose metabolism. Am. J. Physiol. Endocrinol. Metab. 284, E671-E678 (2003).

5. Pedersen, J. S., Andersen, C. B. \& Otzen, D. E. Amyloid structure-one but not the same: the many levels of fibrillar polymorphism. FEBS J. 277, 4591-4601 (2010).

6. Gratzer, W. B. \& Beaven, G. H. Relation between conformation and association state. A study of the association equilibrium of glucagon. J. Biol. Chem. 244, 6675-6679 (1969).

7. Formisano, S., Johnson, M. L. \& Edelhoch, H. Thermodynamics of the selfassociation of glucagon. Proc. Natl. Acad. Sci. USA 74, 3340-3344 (1977). 
8. Johnson, M. L., Formisano, S. \& Edelhoch, H. Self-association of glucagon as measured by the optical properties of rhodamine 6G. J. Biol. Chem. 253, 1353-1356 (1978)

9. Wagman, M. E., Dobson, C. M. \& Karplus, M. Proton NMR studies of the association and folding of glucagon in solution. FEBS Lett. 119, 265-270 (1980).

10. Svane, A. S. P. et al. Early stages of amyloid fibril formation studied by liquid-state NMR: the peptide hormone glucagon. Biophys. J. 95, 366-377 (2008).

11. Pedersen, J. S. et al. The changing face of glucagon fibrillation: structural polymorphism and conformational imprinting. J. Mol. Biol. 355, 501-523 (2006).

12. Andersen, C. B., Otzen, D., Christiansen, G. \& Rischel, C. Glucagon amyloid-like fibril morphology is selected via morphology-dependent growth inhibition. Biochemistry 46, 7314-7324 (2007)

13. Andersen, C. B. et al. Glucagon fibril polymorphism reflects differences in protofilament backbone structure. J. Mol. Biol. 397, 932-946 (2010).

14. Cohen, S. I. A. et al. Proliferation of amyloid- $\beta 42$ aggregates occurs through a secondary nucleation mechanism. Proc. Natl. Acad. Sci. USA 110, 9758-9763 (2013).

15. Estrada, L. C. \& Gratton, E. 3D nanometer images of biological fibers by directed motion of gold nanoparticles. Nano Lett. 11, 4656-4660 (2011).

16. Ferkinghoff-Borg, J. et al. Stop-and-go kinetics in amyloid fibrillation. Phys. Rev. E 82, 010901(R) (2010)

17. Doi, M. \& Edwards, S. F. The theory of polymer dynamics. Oxford University Press, Oxford, 1986

18. Gittes, F., Mickey, B., Nettleton, J. \& Howard, J. Flexural rigidity of microtubules and actin filaments measured from thermal fluctuations in shape. J. Cell. Biol. 120 , 923-934 (1993).

19. Hagerman, P. J. Flexibility of DNA. Annu. Rev. Biophys. Biophys. Chem. 17, 265-286 (1988).

20. van den Akker, C. C., Engel, M. F. M., Velikov, K. P., Bonn, M. \& Koenderink, G. H. Morphology and persistence length of amyloid fibrils are correlated to peptide molecular structure. J. Am. Chem. Soc. 133, 18030-18033 (2011).

21. Oosawa, F. \& Asakura, S. Thermodynamics of the polymerization of protein. Academic Press, Waltham, 1975.

22. Knowles, T. P. J. et al. An analytical solution to the kinetics of breakable filament assembly. Science 326, 1533-1537 (2009).

23. Arosio, P., Beeg, M., Nicoud, L. \& Morbidelli, M. Time evolution of amyloid fibril length distribution described by a population balance model. Chem. Eng. Sci. 78, 21-32 (2012).

24. Hamada, D. \& Dobson, C. M. A kinetic study of $\beta$-lactoglobulin amyloid fibril formation promoted by urea. Protein Sci. 11, 2417-2426 (2002).
25. Cohen, S. I., Vendruscolo, M., Dobson, C. M. \& Knowles, T. J. [The Kinetics and Mechanisms of Amyloid Formation] Amyloid Fibrils and Prefibrillar Aggregates: Molecular and Biological Properties [Otzen, D. E. (ed.)] [183-209] (Wiley-VCH Verlag GmbH \& Co. KGaA, Weinheim, 2013).

26. Orlova, A., Prochniewicz, E. \& Egelman, E. H. Structural dynamics of F-actin: II. Cooperativity in structural transitions. J. Mol. Biol. 245, 598-607 (1995).

27. Kueh, H. Y. \& Mitchison, T. J. Structural plasticity in actin and tubulin polymer dynamics. Science 325, 960-963 (2009).

28. Zhdanov, V. P. Viral capsids: Kinetics of assembly under transient conditions and kinetics of disassembly. Phys. Rev. E 90, 042721 (2014).

\section{Acknowledgments}

This work was supported by the Lundbeck Foundation (BioNET2), the Danish National Research Foundation through the Center for Models of Life and the KU Center of Excellence (MolPhysX). We are grateful to Novo Nordisk A/S for providing glucagon samples.

\section{Author contributions}

M.H.J., L.B.O. and D.O.E. designed the project and supervised the study. P.C. and A.Ky. collected experimental data. P.C., A.Ky., M.H.J., D.E.O. and L.B.O. analyzed the data. A.Ko formulated the model. D.E.O. contributed with reagents. All authors wrote the manuscript.

\section{Additional information}

Competing financial interests: The authors declare no competing financial interests.

How to cite this article: Košmrlj, A. et al. A monomer-trimer model supports intermittent glucagon fibril growth. Sci. Rep. 5, 9005; DOI:10.1038/srep09005 (2015).

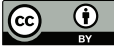

This work is licensed under a Creative Commons Attribution 4.0 International License. The images or other third party material in this article are included in the article's Creative Commons license, unless indicated otherwise in the credit line; if the material is not included under the Creative Commons license, users will need to obtain permission from the license holder in order to reproduce the material. To view a copy of this license, visit http://creativecommons.org/licenses/by/4.0/ 